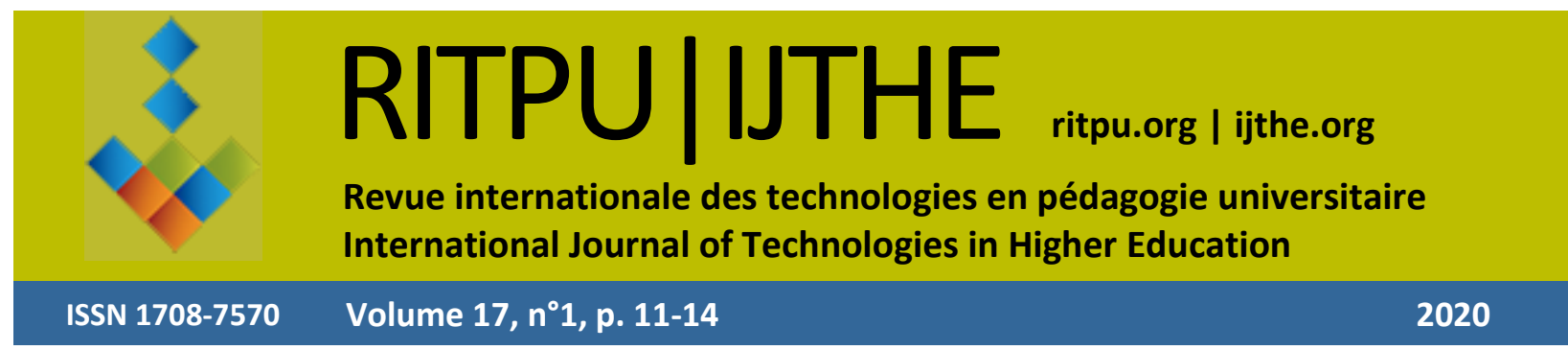

\section{What is the Digital Competency Framework?}

https://doi.org/10.18162/ritpu-2020-v17n1-04
Thierry KARSENTI

thierry.karsenti@umontreal.ca

Bruno POELLHUBER

bruno.poellhuber@umontreal.ca

Simon PARENT

simon.parent.2@umontreal.ca

Florent MICHELOT

florent.michelot@umontreal.ca

Université de Montréal

The Digital Competency Framework is part of the Digital Action Plan (DAP) for Education and Higher Education developed by Québec's Ministère de l'Éducation et de l'Enseignement supérieur (2019). The plan promotes a vision of effective integration and optimal use of digital technologies in order to foster the success of all Quebecers and promote lifelong skills development and maintenance. The first measure of the DAP is structural: the establishment and implementation of a reference framework. The objective was to design an inter-level digital competency framework that spans preschool through higher education, including general education and vocational training. The result is the Digital Competency Framework. It sets out the key dimensions of learning and personal development for 21 st-century learners, teachers, and non-teaching professionals. Elements specific to teaching will be addressed in more detail in an upcoming competency framework for the teaching profession.

The framework is meant to provide the foundation for the development of digital competency. Digital competency is defined as a set of skills necessary to the confident, critical, and creative use of digital technologies to achieve objectives in the areas of learning, work, leisure, and inclusion or participation in society. Structurally, the framework is designed as a continuum of 12 dimensions (see Figure 1 below) that set out the scope of the development of digital competency. The elements of the dimensions are situated on a progress matrix. As they advance through the competency levels, individuals become increasingly autonomous in their use of digital technologies in educational, professional, and everyday life contexts. For example, they are able to select the best digital tools to perform particular tasks.

Digital competency also means that individuals can adapt to technological innovations in years to come (including advances in artificial intelligence), assess these new technologies critically, and adopt and implement those that are useful. 
Finally, digital competency is closely tied to professional development for all 21 st-century workers. Everyone will have to use digital resources in order to keep their professional skills up to date. This will affect communities of practice as well as those taking online training and tutorial programs.

In short, the aim of this framework is to foster the development of digital competency throughout the educational community so that current and future citizens will be autonomous and able to exercise critical judgment in their use of digital technologies. Digital competency is essential in today's world, where technological innovations are transforming the labor market and the skills that employers are looking for. It is therefore vital that teaching practices and programs be adapted to include digital competency in order to prepare future workers for the challenges of tomorrow.

The Digital Competency Framework accounts for the latest research and innovative practices in education (e.g., Karsenti, 2019). Without denying the risks inherent in the so-called digital divide, the framework demonstrates how digital technology can serve as a tool for inclusion. Its Universal Design for Learning (UDL) principle envisages the use of digital technology by all people, without the need for adaptation or special design, and regardless of gender, age, situation, or disability.

The framework adopts a competency-based approach. The term "competency" refers to "complex know-how developed through the effective mobilization of a range of internal and external resources in a variety of related situations" (Tardif, 2007, p. 22). In this sense, competency characteristics are explored as the constituent elements of complex know-how (ibid).

It would be unrealistic, however, to attempt to foresee all possible applications, given the relentless advances in technological developments. All individual competencies draw on numerous and varied resources (ibid), particularly because competencies may evolve over time. Given that we cannot predict the future, our conception of digital competency must be sufficiently flexible and adaptive to prevent invalidation by new technological innovations and digital resources. Consequently, the framework must be able to accommodate technological innovations.

Competencies can also be combined. The framework does not exist in a vacuum. Instead, it allows for a wide range of combinations of resources, be they tools, dimensions, or skills. Furthermore, because competencies are developed over a lifetime (especially digital competencies), a hierarchical or linear framework would be untenable. Each competency may evolve, be reinforced, or be updated, which implies a cyclical nature. Continuous competency development goes hand in hand with the enrichment of one's store of personal resources as one gains experience.

The implementation of this framework is therefore an iterative process. Moreover, the competencies and competency development are fundamentally contextual. Examples drawn from a variety of contexts are provided to illustrate the dimensions of the framework.

The Digital Competency Framework has been shaped by a systematic analysis of more than seventy 21 st-century information literacy and digital competency frameworks from around the world. While it draws on a wealth of existing work, the framework taps into emerging digital trends in education. Compared to documents published in other countries, this project is innovative in its holistic understanding of digital competency. Furthermore, the decision to 
formulate a single digital competency facilitates its integration into other frameworks and documents related to teaching and learning.

The proposed competency is divided into 12 key dimensions (Figure 1). Exercising ethical citizenship in the digital age and Developing and mobilizing technological skills are the central dimensions around which the other dimensions revolve.

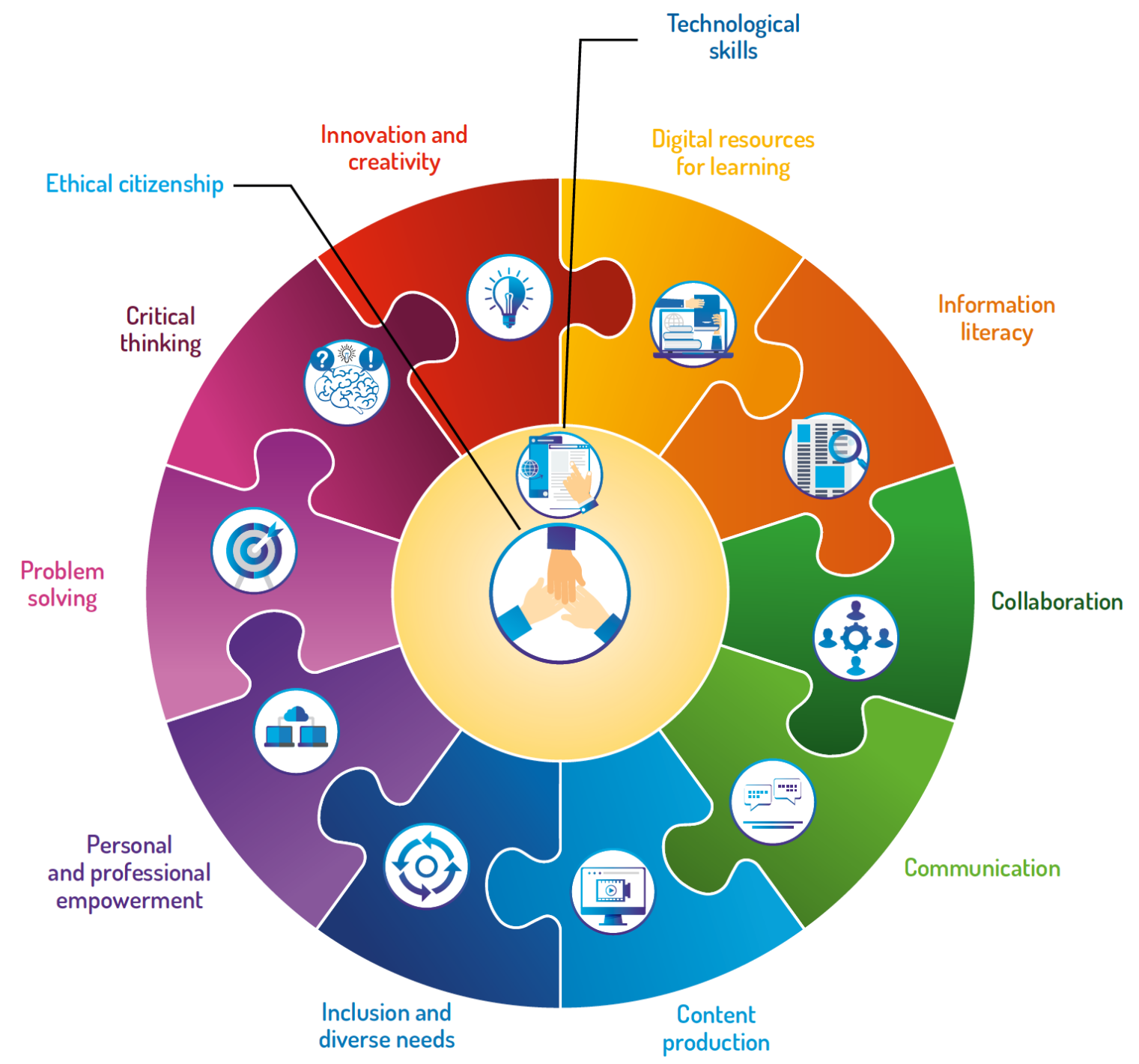

Figure 1

Graphic representation of the 12 dimensions of the Digital Competency Framework

As mentioned above, the framework breaks down digital competency into 12 dimensions, each comprising several elements. In other words, there are three levels to digital competency: competency, dimensions, elements (Figure 2).

The dimensions of the framework are strongly interrelated: they may come into play simultaneously during a given task or they may overlap. A dimension may be reinforced by one or more other dimensions. For instance, an educational activity can be designed to develop both communication skills and collaborative skills through the use of digital technology. The dimensions are therefore numbered in no particular order. 


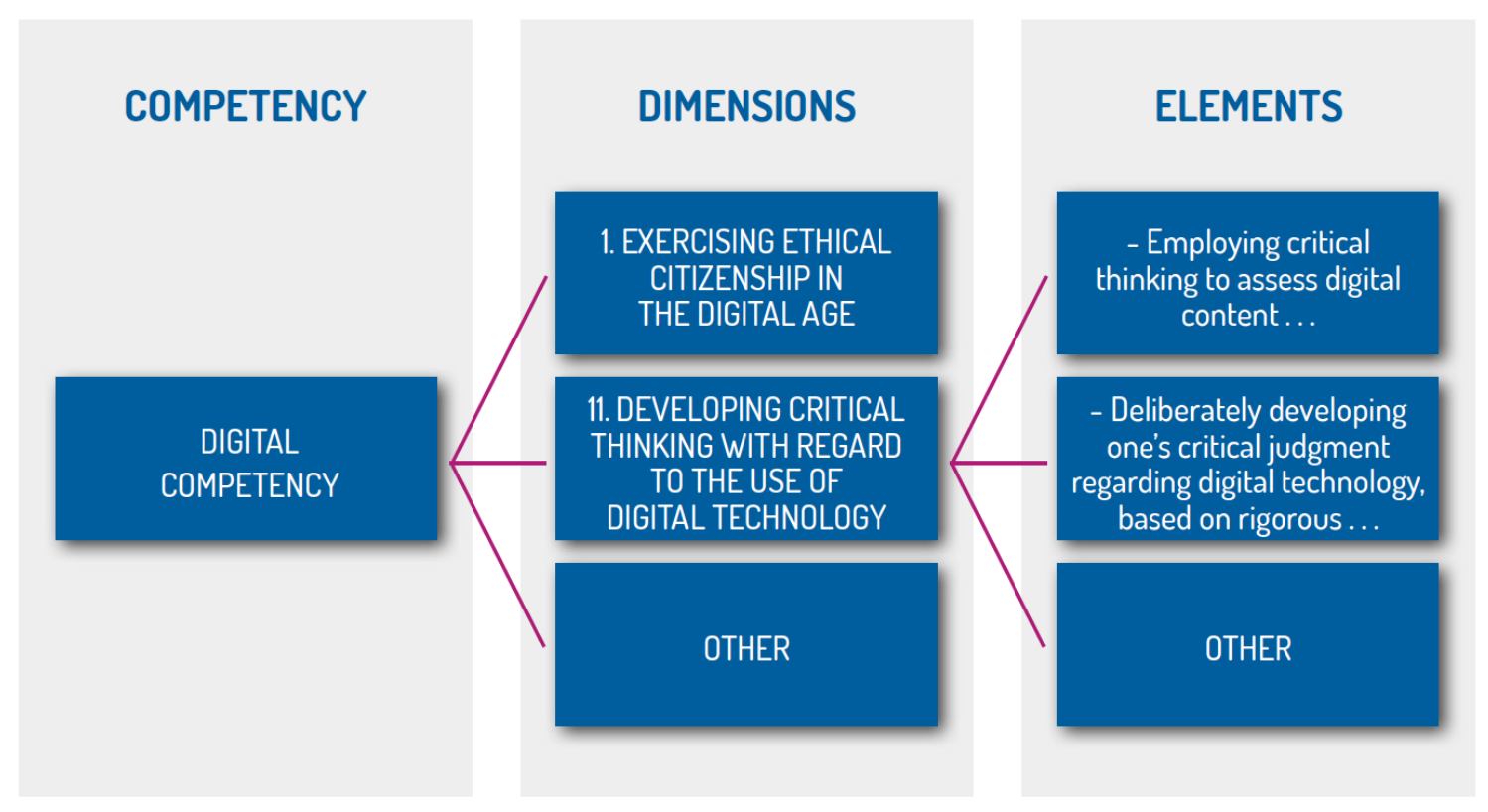

Figure 2.

The structure of the Digital Competency Framework continuum

That said, the first dimension (Exercising ethical citizenship in the digital age) is the cornerstone of the framework, and is generally combined with other dimensions. For example, reflection on ethical considerations regarding copyright (an element of the first dimension) occurs in tandem with the use of information sought and obtained via digital means (an element of the fourth dimension). Similarly, the individual's understanding of digital identity issues (an element of the first dimension) is mobilized in the production and distribution of digital content (an element of the seventh dimension).

The dimensions and their respective elements are not associated with a particular subject, despite initial appearances. Each dimension applies to a multitude of subject-specific contexts. Thus, in order to fully develop digital competency, the different dimensions must be applied in a broad range of contexts.

Each element of the various dimensions begins with an action verb to provide a more concrete understanding of the skills associated with the dimension. All the elements have to do with the use of digital technology, even when this is not explicitly stated. For example, "Mobilizing a variety of resources and solving a problem creatively," an element of the tenth dimension, refers to the ability to use different types of digital technology combined with the ability to think creativity in order to solve problems.

\section{References}

Karsenti, T. (2019). Le numérique en éducation : pour développer des compétences. Presses de l’Université du Québec.

Ministère de l'Éducation et de l'Enseignement supérieur. (2019). Cadre de référence de la compétence numérique. Gouvernement du Québec. http://education.gouv.qc.ca/...

Tardif, J. (2007). L'évaluation des compétences : documenter le parcours de développement. Chenelière Éducation. 